\title{
La competencia comunicativa escrita en la formación de ingenieros
}

\section{The written communicative competence in engineering education}

\author{
DOI: $10.46932 /$ sfjdv2n3-059
}

Received in: May 1st, 2021

Accepted in: Jun 30th, 2021

\author{
Marta Graciela Caligaris \\ Ingeniera Metalúrgica (Facultad Regional San Nicolás, Universidad Tecnológica Nacional) \\ Facultad Regional San Nicolás, Universidad Tecnológica Nacional \\ Colón 332, San Nicolás, Argentina \\ E-mail: mcaligaris@frsn.utn.edu.ar
}

\section{Georgina Beatriz Rodríguez}

Licenciada en Matemática (Facultad de Ciencias Exactas e Ingeniería, Universidad Nacional de Rosario)

Facultad Regional San Nicolás, Universidad Tecnológica Nacional

Colón 332, San Nicolás, Argentina

E-mail: grodriguez@frsn.utn.edu.ar

\section{Lorena Fernanda Laugero}

Licenciada en Enseñanza de la Matemática, (Facultad Regional San Nicolás, Universidad Tecnológica

Nacional)

Facultad Regional San Nicolás, Universidad Tecnológica Nacional

Colón 332, San Nicolás, Argentina

E-mail: llaugero@frsn.utn.edu.ar

\section{RESUMEN}

Las competencias comunicacionales comprenden el conjunto de conocimientos y capacidades que permiten producir y entender mensajes, adecuados a la situación y al contexto. En general, estas competencias conforman un aspecto poco trabajado en la formación de los ingenieros.

El objetivo principal de este trabajo es compartir la experiencia de cátedra desarrollada en Análisis Numérico en la Facultad Regional San Nicolás, Universidad Tecnológica Nacional, Argentina, con el fin de trabajar la competencia de comunicación escrita.

Dentro de esta experiencia se realizaron distintas instancias de evaluación para medir los resultados del trabajo realizado, que también son presentados. El análisis de los mismos permite concluir que la aplicación de esta metodología de trabajo contribuyó notoriamente a mejorar la competencia comunicativa escrita de los estudiantes.

Palabras Clave: competencias, comunicación escrita, formación ingenieril

Communication skills include the set of knowledge and abilities that allow producing and understanding messages, appropriate to the situation and context. Usually, these skills constitute a little worked aspect in the training of engineers.

The main objective of this work is to share the experience developed in Numerical Analysis at the Facultad Regional San Nicolás, Universidad Tecnológica Nacional, Argentina, in order to work on the written communication competence.
} 
Within this experience, different assessments instances were carried out to measure the results of the performed work, and are also presented. Their analysis allows to conclude that the application of this work methodology contributed significantly to improving students' written communicative competence.

Keywords: competencies, written communication, engineering training

\title{
1 INTRODUCCIÓN
}

El antiguo paradigma de formación de profesionales basado en la enseñanza como simple esquema de transferencia de conocimientos que el alumno oportunamente sabrá abstraer, articular y aplicar, ha ido perdiendo espacio en la realidad actual. La sociedad de hoy requiere de egresados universitarios competentes, capaces de ejercer su profesión en la realidad que los rodea [1]. Tal como sostienen Arana Ercilla y Batista Tejeda [2]:

\begin{abstract}
La búsqueda de una cultura integral es un objetivo estratégico en el mundo de hoy, así un humanista que no sepa de los avances científicos tecnológicos puede poseer una elevada cultura humanista y ser un ignorante ante las nuevas formas de vida imperantes. De la misma manera, un científico o un tecnólogo que posea elevados conocimientos y habilidades profesionales, tiene que saber conducirlas desde y para la sociedad, lo que se expresa en saber trabajar en grupo, interpretar social y económicamente las necesidades y demandas; dirigir procesos a través de la participación, el diálogo y la comunicación, en busca de información valiosa para la competitividad. (p.13)
\end{abstract}

Esta situación hace que sea necesario definir un nuevo modelo de competencias profesionales. Así es como el Consejo Federal de Decanos de Facultades de Ingeniería (CONFEDI) de la República Argentina comienza a trabajar para alcanzar un acuerdo sobre las competencias que se deberían desarrollar en los graduados de ingeniería en Argentina. En 2006, después del trabajo realizado durante dos años por una comisión creada para tal fin, se suscribió por votación unánime del plenario de decanos, el documento que sintetiza las Competencias Genéricas de Egreso del Ingeniero Argentino. Este acuerdo orienta a las facultades de ingeniería en la definición de sus procesos de enseñanza y aprendizaje tendientes al desarrollo de competencias en sus alumnos [3]. La clasificación de las competencias genéricas de egreso dada por el CONFEDI presenta la siguiente estructura:

\section{- Competencias tecnológicas.}

a) Identificar, formular y resolver problemas de ingeniería.

b) Concebir, diseñar y desarrollar proyectos de ingeniería.

c) Gestionar, planificar, ejecutar y controlar proyectos de ingeniería.

d) Utilizar de manera efectiva las técnicas y herramientas de aplicación en la ingeniería.

e) Contribuir a la generación de desarrollos tecnológicos y/o innovaciones tecnológicas. 


\section{- Competencias sociales, políticas y actitudinales.}

a) Desempeñarse de manera efectiva en equipos de trabajo.

b) Comunicarse con efectividad.

c) Actuar con ética, responsabilidad profesional y compromiso social, considerando el impacto económico, social y ambiental de su actividad en el contexto local y global.

d) Aprender en forma continua y autónoma.

e) Actuar con espíritu emprendedor.

La competencia comunicativa consiste en la capacidad de construir enunciados que no solo sean gramaticalmente correctos, sino también socialmente apropiados [4]. Es decir, implica un conjunto de conocimientos, procesos y experiencias de diversos tipos que el emisor - receptor deberá poner en juego para producir o comprender discursos adecuados a la situación y al contexto de comunicación [5].

En general, la competencia comunicativa es un aspecto poco trabajado en la formación de los futuros ingenieros. Una de las principales causas puede ser que aquellas disciplinas tradicionalmente relacionadas con la ingeniería se desarrollan explícitamente; mientras que las áreas que fomentan la formación integral de un ingeniero, sólo se trabajan de manera general.

Con la finalidad de trabajar la competencia comunicativa escrita con los alumnos que cursaron Análisis Numérico en la Facultad Regional San Nicolás (FRSN) en las especialidades Ingeniería Mecánica y Electrónica en el ciclo 2019, se evaluó el nivel de desarrollo de dicha competencia al inicio del cursado. Para ello, se les propuso una actividad donde debían explicar o justificar ciertas situaciones apelando al marco teórico desarrollado.

Los resultados obtenidos pusieron de manifiesto las dificultades y carencias que presentan los alumnos en relación a la expresión escrita, en particular para explicar cuestiones relacionadas con la matemática [6].

Con el propósito de afianzar dicha competencia en los estudiantes, en los restantes temas desarrollados se incluyeron actividades donde los alumnos debían dar una fundamentación de la respuesta.

Para analizar el grado de avance de la competencia comunicativa en los alumnos, se propuso la realización de un trabajo práctico en una de las últimas unidades. En este trabajo práctico, debían elaborar un texto de al menos 150 palabras. Las producciones realizadas por los estudiantes fueron corregidas a partir de una rúbrica que se elaboró considerando algunas propiedades textuales.

El objetivo principal de este trabajo es compartir la experiencia de cátedra desarrollada. También, se muestran los resultados obtenidos en cada una de las instancias y algunas conclusiones extraídas a partir de los datos obtenidos. 
Para una mejor organización, este artículo está constituido por cinco secciones. En la segunda sección, se plantea el marco teórico de la investigación. En la tercera, se presenta la experiencia de cátedra realizada. Se realiza una breve descripción de los participantes, se muestran las actividades con las cuales se trabajó, las propiedades textuales que se consideraron para analizar cada una de las producciones de los alumnos y la forma en que fue procesada la información obtenida. En la cuarta sección, se muestran los resultados obtenidos y por último, en la quinta sección, se presentan las conclusiones sobre la experiencia realizada.

\section{CONCEPTO DE COMPETENCIA}

Existen diversas definiciones del concepto de competencia. Así, Gerard y Roegiers [7] consideran que las competencias están dadas por un conjunto integrado de capacidades que permite, de manera espontánea, comprender una situación y responder a ella de manera más o menos pertinentemente. Le Boterf [8] las define como un saber actuar, es decir un saber integrar, movilizar y transferir un conjunto de recursos (conocimientos, saberes, aptitudes, razonamientos) en un contexto dado para enfrentar diferentes problemas o para realizar una tarea. Por su parte, el CONFEDI [9] sostiene que una competencia es la capacidad de articular eficazmente un conjunto de esquemas (estructuras mentales) y valores, permitiendo movilizar (poner a disposición) distintos saberes, en un determinado contexto con el fin de resolver situaciones profesionales. Esta definición señala que las competencias:

- aluden a capacidades complejas e integradas.

- $\quad$ están relacionadas con saberes (teórico, contextual y procedimental).

- $\quad$ se vinculan con el saber hacer (formalizado, empírico, relacional).

- $\quad$ están referidas al contexto y desempeño profesional.

- permiten incorporar la ética y los valores.

\subsection{LAS COMPETENCIAS GENÉRICAS DE EGRESO}

Según el CONFEDI, hay consenso en cuanto que el ingeniero no sólo debe saber, sino también saber hacer. El saber hacer no surge de la mera adquisición de conocimientos sino que es el resultado de la puesta en funciones de una compleja estructura de conocimientos, habilidades y destrezas que requiere 
ser reconocida expresamente en el proceso de aprendizaje para que la propuesta pedagógica incluya las actividades que permitan su desarrollo [9].

El diseño por competencias ayuda a vigorizar el saber hacer requerido a los ingenieros recién recibidos. En este sentido, la formación de grado debe desarrollar aquellas competencias que el recién graduado tiene que poseer, en un nivel de desarrollo adecuado, para una efectiva inserción laboral.

El CONFEDI contempla diez competencias genéricas, complejas e integradas, relacionadas con saberes (teórico, contextual y procedimental), que se vinculan con el saber hacer (formalizado, empírico, relacional), que están referidas al contexto profesional (la situación en que el profesional debe desempeñarse o ejercer), que apuntan al desempeño profesional (la manera en que actúa un profesional técnicamente competente y socialmente comprometido) y que incorporan la ética y los valores en el perfil del profesional que se busca formar.

\subsection{LA COMPETENCIA COMUNICATIVA}

Una de las competencias genéricas de egreso propuesta para los ingenieros argentinos es la de comunicarse con efectividad [9].

Según Labrador y Morote [10], la competencia comunicativa es la capacidad para transmitir conocimientos, expresar ideas y argumentos de manera clara, rigurosa y convincente, tanto en forma oral como escrita, utilizando los recursos y medios adecuados, en función de las características de la situación y de la audiencia.

Estas autoras sostienen también que las diversas situaciones con las cuales se enfrenta el alumno en su vida académica y las que afrontarán en su futuro profesional, llevan a considerar a la competencia comunicativa como una competencia relevante, ya que el estudiante participa en el aula para expresar dudas, para responder preguntas, así como también, para realizar trabajos.

Con la finalidad de poder desarrollar esta competencia, es necesario que el alumno posea un cierto grado de confianza en sí mismo y adaptación al entorno para poder realizar una comunicación eficiente.

Con respecto a la matemática, Whitin y Whitin [11] señalan que la comunicación no siempre es reconocida como algo importante en su proceso de enseñanza. No obstante, Silbey sostiene que una de las claves para la profundización de la comprensión matemática se basa en la comunicación [12]. Así, por ejemplo, que los estudiantes hablen de un problema, que escriban los pasos para resolverlo, que escuchen las soluciones de sus compañeros, los ayuda a estructurar y consolidar su pensamiento matemático o adquirir el vocabulario específico de la materia. 


\subsection{LA COMPETENCIA COMUNICATIVA ESCRITA}

$\mathrm{Si}$ se desea preparar a los fututos ingenieros con los conocimientos esperados para su perfil profesional, es necesario incluir en los planes de estudio la cultura escrita para que, de esta manera, puedan desempeñarse como profesionales del área a la que pertenecen [13].

Para que un estudiante desarrolle la competencia comunicativa escrita es necesario exponerlo a prácticas que permitan el desarrollo de tal competencia.

\section{EXPERIENCIA DE CÁTEDRA}

Para desarrollar o afianzar la competencia comunicativa escrita en los alumnos que cursaron Análisis Numérico en el ciclo 2019, se diseñaron diversas actividades donde los estudiantes debían justificar, fundamentar, explicar ciertas situaciones apelando al marco teórico desarrollado.

En las siguientes subsecciones, se indican los aspectos más importantes de la experiencia de cátedra realizada.

\subsection{DESCRIPCIÓN DE LOS PARTICIPANTES}

Para llevar adelante esta experiencia de cátedra, se seleccionó como grupo de estudio a los alumnos de las carreras Ingeniería Mecánica y Electrónica que cursaron Análisis Numérico durante el ciclo 2019.

Si bien, inicialmente, la población total estaba conformada por cuarenta y nueve alumnos, trece estudiantes no fueron considerados por no haber cumplimentado con las exigencias que se establecen para alcanzar la condición de “alumno regular". Los diecisiete alumnos de Ingeniería Electrónica y los diecinueve alumnos de Ingeniería Mecánica que alcanzaron la condición de regular en las respectivas asignaturas fueron considerados como partícipes de la experiencia.

\subsection{ACTIVIDADES PROPUESTAS}

A continuación, se muestran las actividades que se le plantearon a los alumnos tanto al inicio del cursado como al inicio del segundo cuatrimestre. También se presentan las situaciones problemáticas que se elaboraron para afianzar o superar algunas de las dificultades que se detectaron, en cuanto a la competencia comunicativa, durante el aprendizaje de los métodos numéricos.

\subsection{ACTIVIDAD PROPUESTA AL INICIO DEL CURSADO DE LA MATERIA.}

Al finalizar la enseñanza del primer tema que se trata en la materia, se propuso a los alumnos de ambos cursos la actividad que se muestra en la Fig. 1. 
Figura 1. Actividad propuesta para evaluar la competencia comunicativa al finalizar el desarrollo de la unidad "Resolución de ecuaciones no lineales".

La siguiente tabla muestra las ocho primeras aproximaciones obtenidas al resolver
la ecuación $x^{8}-1=0$ por medio del método de Regula Falsi, tomando como
intervalo inicial $[0,5 ; 1,5]$.
\begin{tabular}{|c|c|}
\hline Iteración & Aproximación \\
\hline 1 & 0.53887 \\
\hline 2 & 0.57612 \\
\hline 3 & 0.61175 \\
\hline 4 & 0.64575 \\
\hline 5 & 0.67811 \\
\hline 6 & 0.70880 \\
\hline 7 & 0.73778 \\
\hline 8 & 0.76500 \\
\hline
\end{tabular}
a) ¿Qué es lo que sucede cuando se aplica el método? ¿Por qué?
b) ¿Cómo solucionaría el inconveniente para mejorar la eficiencia del método?
Ejemplificar.

Para la realización de la actividad planteada, los estudiantes debían explicar, ejemplificar y justificar apelando a los distintos conceptos aprendidos en la unidad.

\subsection{ACTIVIDADES DISEÑADAS PARA PROMOVER LA COMPETENCIA COMUNICATIVA}

Con el objetivo de realizar las intervenciones pertinentes y promover el desarrollo de la competencia comunicativa escrita, se diseñaron distintas actividades en cada una de las siguientes unidades tratadas. A modo de ejemplo, en las Fig. 2 - 5, se muestran algunas de ellas.

Figura 2. Actividades propuestas durante el aprendizaje de los métodos para resolver sistemas de ecuaciones lineales.

\begin{tabular}{|c|c|c|c|c|}
\hline \multicolumn{4}{|c|}{$\begin{array}{l}\text { Sea el sistema de ecuaciones }\left(\begin{array}{ccc}5 & -1 & 2 \\
-1 & 6 & -2 \\
2 & -2 & 8\end{array}\right) \cdot\left(\begin{array}{l}x_{1} \\
x_{2} \\
x_{3}\end{array}\right)\left(\begin{array}{l}6 \\
3 \\
8\end{array}\right) \\
\text { ¿Se podrá aplicar cualquiera de los dos métodos iterativos estudiados si se sabe } \\
\text { que } \rho\left(T_{J}\right) \approx 0,53 \text { ? ¿Por qué? }\end{array}$} & \\
\hline & & $\begin{array}{l}\text { Sea el sistema de ecuaciones } \\
\text { ¿Cuál es el método más aprc } \\
\text { la matriz de coeficientes son } \\
\text { Fundamentar la respuesta. }\end{array}$ & $\left.\begin{array}{lccc}2 & 5 & -2 & 0 \\
0 & -2 & 5 & 2 \\
0 & 0 & 2 & 5\end{array}\right)$ & $\begin{array}{l}\left(\begin{array}{l}x_{2} \\
x_{1} \\
x_{4}\end{array}\right)=\left(\begin{array}{c}9 \\
-3 \\
-3\end{array}\right) \\
\text { plicar? Se sabe que los autovalores de } \\
\beta_{2} \approx 3,764, \beta_{3} \approx 6,236 \text { y } \beta_{4} \approx 8,236 \text {. }\end{array}$ \\
\hline
\end{tabular}


Figura 3. Actividades propuestas durante el aprendizaje de los métodos de interpolación y ajuste de curvas.

Para aproximar la función $f(x)=\sqrt[3]{x^{2}}$ se construyó el polinomio de interpolación de Lagrange utilizando, en un caso, 11 puntos equiespaciados, mientras que en el otro, las raíces del polinomio de Chebyshev de grado 11.

a) ¿De qué grado es el polinomio de interpolación obtenido en ambos casos?

b) Indicar qué gráfica le corresponde al polinomio de interpolación tomando puntos equiespaciados. ¿Por qué?

c) ¿Cuál de las dos opciones es la más adecuada para obtener una aproximación de $f(1,9)$ ? Justificar la respuesta.

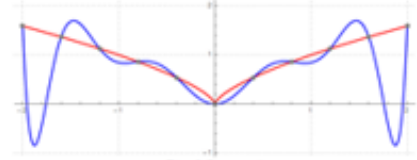

Gráfica A

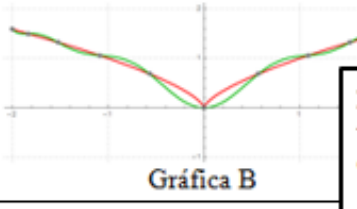

ley que vincula las variables $x$ y $T$ establece que $T=k x$. Explicar qué método utilizaría para dar una aproximación de la constante $k$, a partir del siguiente conjunto de datos.

Figura 4. Actividades propuestas durante el aprendizaje de los métodos de integración numérica.

Se aplicaron las reglas de los trapecios y de Simpson para resolver la integral:

$$
\int_{0}^{1} 2 \cdot e^{2 x} \cdot d x
$$

utilizando siete evaluaciones de la función. La siguiente tabla muestra las soluciones numéricas obtenidas. Explicar qué regla se aplicó en cada caso. Justificar la respuesta.

\begin{tabular}{|c|c|c|}
\hline Solución exacta & Regla 1 & Regla 2 \\
\hline 6,389056099 & 6,3894886 & 6,4481048 \\
\hline
\end{tabular}

Se aplicó una cuadratura de Gauss a la siguiente integral: $\int_{-1}^{1}\left[x^{4}+5\right] \cdot d x=10,4$.

La siguiente tabla muestra las soluciones numéricas obtenidas. Explicar qué es lo que sucede en cada caso.

\begin{tabular}{|c|c|}
\hline Cantidad de puntos & Cuadratura de Gauss \\
\hline 2 & 10.222222 \\
\hline 3 & 10.400001 \\
\hline
\end{tabular}

Figura 5. Actividades propuestas durante el aprendizaje de los métodos para resolver ecuaciones diferenciales ordinarias.

$\mathrm{La}$ siguiente tabla muestra los resultados que se obtuvieron al aproximar la derivada primera de la función $f(x)=\operatorname{sen}(x)$ en $x=1$ utilizando como tamaño de paso $h=0,5$. Indicar y fundamentar en cada caso, cuál es el método que se aplicó (diferencias regresivas, diferencias centradas).

\begin{tabular}{|c|c|c|}
\hline Método & Aproximación & Error absoluto \\
\hline 1 & 0,5181 & 0,0222 \\
\hline 2 & 0,7241 & 0,1838 \\
\hline
\end{tabular}

Se aplicó el método de Euler, Taylor de orden dos y Runge - Kutta de orden cuatro al problema de valor inicial $y^{\prime}=2 \cdot y+x-1$ con $y(0)=5$. La siguiente gráfica muestra las soluciones numéricas obtenidas utilizando el mismo tamaño de paso. Indicar a qué método corresponde cada una de las soluciones discretas. Justificar las elecciones realizadas.

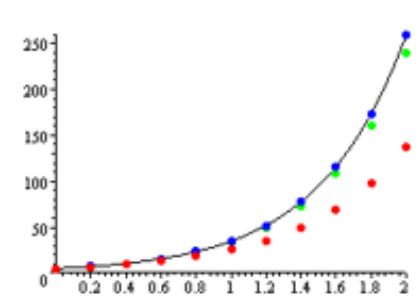




\subsection{ACTIVIDAD PROPUESTA PARA EVALUAR EL NIVEL DE DESARROLLO DE LA} COMPETENCIA COMUNICATIVA

Con el objetivo de analizar el resultado de la realización de las actividades, como las mostradas en la subsección anterior, y también estimar del grado de desarrollo de la competencia comunicativa escrita en los alumnos, se les propuso la consigna que se muestra en la Fig. 6.

La actividad planteada fue resuelta por los estudiantes una vez finalizado el tema "Resolución de problemas de valor inicial en una dimensión", tema que se desarrolla en una de las últimas unidades de la materia.

Figura 6. Actividad propuesta al finalizar el desarrollo de la unidad "Resolución de problemas de valor inicial en una dimensión".

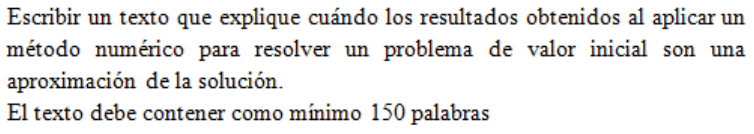

\subsection{PROPIEDADES TEXTUALES}

Estudios de la lingüística textual y de la gramática del discurso sostienen que cuando una persona habla o escribe se construyen textos, y para hacerlo, es necesario dominar ciertas habilidades que tienen que ver con las propiedades textuales. Estas habilidades, por ejemplo, hacen referencia a la capacidad para seleccionar las palabras adecuadas, conectar frases entre sí, usar correctamente las reglas ortográficas, de puntuación o morfosintácticas [14]. Para analizar las producciones realizadas por los estudiantes, se tomaron en cuenta las propiedades textuales que se muestran en la Tabla 1.

Tabla 1. Propiedades textuales consideradas para el análisis de los textos.

\begin{tabular}{|l|l|}
\hline Propiedad textual & Indicadores \\
\hline $\begin{array}{l}\text { Adecuación } \\
\text { (adaptación del texto a la situación comunicativa) }\end{array}$ & $\begin{array}{l}\text { Intención comunicativa (finalidad que se pretende: informar, } \\
\text { opinar, convencer...) } \\
\text { Enfoque del tema (tratamiento que se le quiere dar a la } \\
\text { información que se transmite: general, específico, divulgativo, } \\
\text { técnico...) }\end{array}$ \\
\hline $\begin{array}{l}\text { Cantidad de información (discriminar la información } \\
\text { transmisión de la información de una manera } \\
\text { clara, ordenada y comprensible) }\end{array}$ & $\begin{array}{l}\text { lógico al destinatario) } \\
\text { Estructuración de la información (organización del } \\
\text { discurso para evitar desorden de ideas) } \\
\text { Calidad de la información (redacción del texto con lógica y } \\
\text { congruencia). }\end{array}$ \\
\hline $\begin{array}{l}\text { Empleo correcto de los signos de puntuación. } \\
\text { Cohesión } \\
\text { (relación correcta de las ideas desde el punto de de conectores de discurso para relacionar ideas } \\
\text { vista del léxico y de la gramática) } \\
\text { (conectores de tiempo, espacio, de ejemplificación, } \\
\text { explicativos, de causa, de consecuencia...) } \\
\text { Repeticiones innecesarias de palabras. } \\
\text { Uso adecuado del tiempo y modo verbal. }\end{array}$ \\
\hline
\end{tabular}




\begin{tabular}{|l|l|}
\hline & $\begin{array}{l}\text { Utilización apropiada de las relaciones de concordancia } \\
\text { gramatical (concordancia entre sujeto - verbo y entre } \\
\text { sustantivo-adjetivo). }\end{array}$ \\
\hline $\begin{array}{l}\text { Corrección } \\
\text { (respeto de las normas que rigen el uso correcto de } \\
\text { la lengua) }\end{array}$ & $\begin{array}{l}\text { Utilización correcta del léxico. } \\
\text { Empleo adecuado de las reglas ortográficas y } \\
\text { gramaticales. }\end{array}$ \\
\hline
\end{tabular}

\subsection{PROCESAMIENTO DE LA INFORMACIÓN}

A partir de los distintos indicadores considerados, se utilizó una escala para determinar el grado de desarrollo de cada uno de ellos. La Tabla 2 muestra la escala utilizada y el valor numérico que se le asignó a cada una de las opciones.

Tabla 2. Escala utilizada y su valor numérico

\begin{tabular}{|l|l|}
\hline Escala & Valor numérico \\
\hline Muy bueno & 4 \\
\hline Bueno & 3 \\
\hline Regular & 2 \\
\hline Deficiente & 1 \\
\hline No resuelve & 0 \\
\hline
\end{tabular}

Con el objetivo de realizar una rápida lectura de los resultados obtenidos por los estudiantes en cada una de las actividades, se acudió a la aplicación de herramientas provenientes de la estadística descriptiva. Por medio de la construcción de tablas de frecuencias, gráficos porcentuales y cálculo de parámetros de tendencia central, se sintetizó la información relevada.

\section{RESULTADOS Y DISCUSIÓN}

Las Fig. 7 - 10 muestran los resultados obtenidos al tabular la información obtenida en cada una de las actividades que se analizaron durante el aprendizaje de los métodos numéricos.

\subsection{RESULTADOS DE LA ACTIVIDAD SOBRE "RESOLUCIÓN DE ECUACIONES NO LINEALES"}


Figura 7. Resultados de la actividad propuesta al finalizar el desarrollo de la unidad "Resolución de ecuaciones no lineales" en Ingeniería Mecánica.

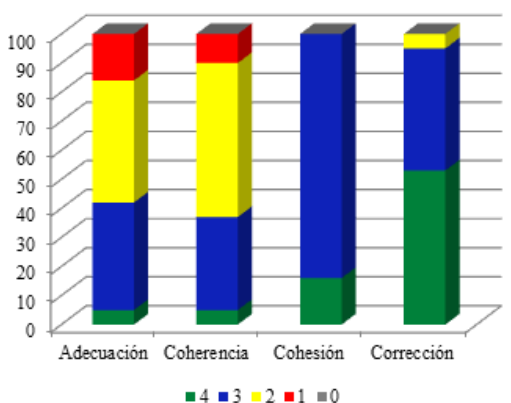

Como se puede observar en la Fig. 7, las mayores dificultades que presentaban los alumnos de Ingeniería Mecánica al inicio del ciclo 2019 estaban vinculadas con las propiedades textuales de adecuación y coherencia. Aproximadamente, sólo el $40 \%$ de los estudiantes demostró de manera muy buena o buena estas propiedades. Esta situación probablemente ocurre porque muchos alumnos no podían adaptar correctamente el texto a la situación comunicativa en la que se desarrollaba ni transmitir la información de una manera clara y ordenada.

No obstante, con respecto a la cohesión y corrección, alrededor del 95\% de los estudiantes no presentó inconvenientes con estas propiedades. Muy pocos estudiantes presentaron dificultades con el empleo correcto de los signos de puntuación y de las reglas ortográficas y gramaticales.

Figura 8. Resultados de la actividad propuesta al finalizar el desarrollo de la unidad "Resolución de ecuaciones no lineales" en Ingeniería Electrónica.

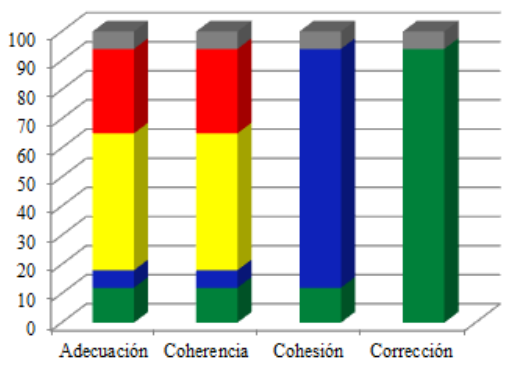

$-4=3=2=1=0$

En cambio, en Ingeniería Electrónica, como se puede apreciar en la Fig. 8, la situación resultó ser mucho más crítica ya que el $82 \%$ de los estudiantes presentó dificultades con la propiedad textual de coherencia. Uno de los principales inconvenientes es la incapacidad para estructurar de manera adecuada la información para evitar el desorden de ideas. También el $82 \%$ tuvo inconvenientes con la propiedad de adecuación. Es decir, presentó dificultades para transmitir de manera apropiada la finalidad que se pretende con el texto. 
Análogamente a lo ocurrido en el grupo de Ingeniería Mecánica, los alumnos de Ingeniería Electrónica tampoco presentaron falencias en cuanto a los distintos indicadores de las propiedades de cohesión y corrección. 
Figura 9. Resultados de la actividad propuesta al finalizar el desarrollo de la unidad "Resolución de problemas de valor inicial en una dimensión" en Ingeniería Mecánica.

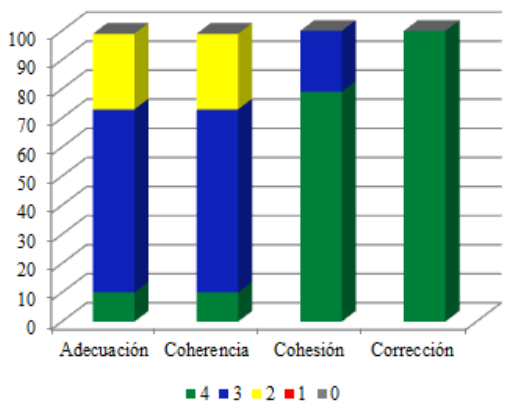

En la Fig. 9 se representan los resultados obtenidos por los estudiantes de Ingeniería Mecánica en la actividad sobre "Resolución de problemas de valor inicial en una dimensión", uno de los últimos temas desarrollados en la asignatura.

A partir de la observación del gráfico, se puede deducir que hubo una mejora notoria en los resultados obtenidos, dado que el $72 \%$ de los estudiantes logró elaborar un texto con buena o muy buena adecuación y coherencia. Además, la totalidad de los alumnos pudo emplear correctamente el vocabulario específico del tema, así como también las reglas ortográficas y gramaticales (propiedad textual de corrección).

Sólo el $21 \%$ de los estudiantes presentó pequeñas dificultades en cuanto al uso correcto de los signos de puntuación.

Figura 10. Resultados de la actividad propuesta al finalizar el desarrollo de la unidad "Resolución de problemas de valor inicial en una dimensión” en Ingeniería Electrónica.

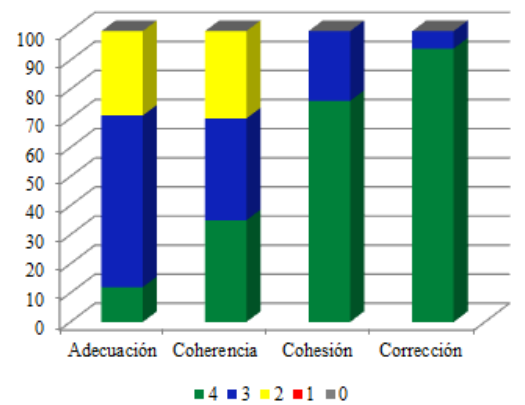

Una situación similar ocurrió con los alumnos de Ingeniería Electrónica debido a que únicamente el 30\% de los estudiantes demostró de manera regular las propiedades textuales de adecuación y 
coherencia. En cambio, con respecto a la cohesión y corrección, ningún estudiante presentó dificultades importantes en dichas propiedades.

Una posible causa de la mejora en ambos cursos fue el afianzamiento que lograron los alumnos por medio de las actividades propuestas por las docentes a cargo de la cátedra en los distintos temas que se desarrollaron. Otro factor que pudo haber influido es el hecho que esta consigna, a diferencia de la actividad inicial planteada, fue resuelta por los estudiantes fuera del horario de clase, por lo cual tuvieron más tiempo para elaborar un texto que cumpliera con todas las propiedades textuales consideradas.

\subsection{ANÁLISIS DEL IMPACTO DE LA METODOLOGÍA APLICADA}

Con el objetivo de cuantificar el impacto que tuvo la realización de actividades para afianzar o fortalecer la competencia comunicativa escrita en los alumnos, se calculó para cada una de las propiedades textuales de las actividades analizadas, un promedio teniendo en cuenta el valor numérico asignado a cada categoría de la escala considerada. Las Tablas 3 y 4 muestran los promedios obtenidos.

A partir de la comparación de las tablas correspondientes a la misma especialidad, se puede concluir que en todos los casos los promedios correspondientes a las distintas propiedades textuales se incrementaron.

En la especialidad Ingeniería Mecánica, el incremento en cada uno de los promedios se produjo en igual medida para las distintas propiedades textuales (aproximadamente hubo un incremento del 0,55 en cada uno de los promedios). En cambio, en Ingeniería Electrónica, los incrementos se produjeron en distinta proporción. Así, por ejemplo, el promedio correspondiente a la propiedad textual coherencia aumentó su valor 1,18 puntos mientras que el de corrección, sólo en 0,18 puntos.

Tabla 3. Promedios obtenidos en cada una de las propiedades textuales en la actividad correspondiente a "Resolución de ecuaciones no lineales".

\begin{tabular}{|l|l|l|l|l|}
\cline { 2 - 5 } \multicolumn{1}{c|}{} & \multicolumn{3}{l|}{ Propiedades textuales } \\
\cline { 2 - 5 } \multicolumn{1}{c|}{} & Adecuación & Coherencia & Cohesión & Corrección \\
\hline Ingeniería Mecánica & 2,32 & 2,32 & 3,16 & 3,47 \\
\hline Ingeniería Electrónica & 1,88 & 1,88 & 2,94 & 3,76 \\
\hline
\end{tabular}

Tabla 4. Promedios obtenidos en cada una de las propiedades textuales en la actividad correspondiente a "Resolución de problemas de valor inicial en una dimensión".

\begin{tabular}{|l|l|l|l|l|}
\cline { 2 - 5 } \multicolumn{1}{c|}{} & \multicolumn{3}{l|}{ Propiedades textuales } \\
\cline { 2 - 5 } \multicolumn{1}{c|}{} & Adecuación & Coherencia & Cohesión & Corrección \\
\hline Ingeniería Mecánica & 2,84 & 2,84 & 3,79 & 4 \\
\hline Ingeniería Electrónica & 2,82 & 3,06 & 3,76 & 3,94 \\
\hline
\end{tabular}

Otra cuestión para destacar es que, si bien en ambas especialidades hubo un incremento en los promedios de las propiedades textuales, en la especialidad Ingeniería Electrónica, éste fue mayor. Como se puede apreciar en la Tabla 5, el promedio general de las propiedades textuales correspondiente a la 
especialidad Ingeniería Mecánica inicialmente era superior. Sin embargo, esta situación se revertió en la actividad correspondiente a "Resolución de problemas de valor inicial en una dimensión". Estos resultados permiten inferir que el impacto que produjo la aplicación de la metodología empleada durante el aprendizaje de los métodos numéricos fue menor en Ingeniería Mecánica que en Ingeniería Electrónica. Un factor que puede haber influido, al menos en la situación inicial, es el hecho de que los alumnos de Ingeniería Electrónica son estudiantes del segundo nivel de la carrera mientras que los de Ingeniería Mecánica, pertenecen al tercer nivel.

Tabla 5. Promedios generales de las propiedades textuales en cada una de las actividades analizadas según la especialidad de los alumnos.

\begin{tabular}{|l|l|l|}
\cline { 2 - 3 } \multicolumn{1}{c|}{} & \multicolumn{2}{l|}{ Especialidad } \\
\hline Actividad & Mecánica & Electrónica \\
\hline $\begin{array}{l}\text { Resolución de ecuaciones } \\
\text { no lineales }\end{array}$ & $\mathbf{2 , 8 2}$ & $\mathbf{2 , 6 2}$ \\
\hline $\begin{array}{l}\text { Resolución de problemas } \\
\text { de valor inicial en una } \\
\text { dimensión }\end{array}$ & $\mathbf{3 , 3 7}$ & $\mathbf{3 , 4 0}$ \\
\hline
\end{tabular}

\section{CONCLUSIONES}

En el mundo laboral actual, la competencia comunicativa es fundamental para un adecuado desempeño profesional. Por esta razón, uno de los grandes retos que tiene la educación superior, se basa en desarrollar esta competencia de manera adecuada en los futuros profesionales. Tal como sostienen Kindelán y Martín [15]:

Las destrezas comunicativas son, pues, indispensables para transmitir los conocimientos y la información, intercambiar ideas y hacer llegar los resultados del trabajo a un público determinado. Cada vez más empresarios necesitan reclutar trabajadores que sean capaces de utilizar estas destrezas en todos los niveles jerárquicos de una organización o empresa a fin de lograr un trabajo en equipo coordinado y eficaz; trabajadores que posean habilidades y destrezas de un alto nivel como el análisis, la síntesis, la crítica constructiva y la capacidad de interacción con otras partes interesadas, todo ello con el único fin de hacer progresar la organización. Por tanto, las destrezas comunicativas han de ser objetivo inmediato de la formación académica y profesional del individuo en este nuevo siglo. (p. 733)

Los docentes a cargo de la cátedra Análisis Numérico, con el objetivo de enseñar los contenidos de la asignatura y colaborar con el desarrollo de las competencias genéricas de egreso, aplicaron para el dictado de la materia una nueva metodología de enseñanza.

En particular, al inicio del ciclo 2019, observaron que las producciones escritas de sus alumnos no eran de buena calidad debido a que las mismas presentaban muchos errores de adecuación y coherencia. Esta situación hizo que en el desarrollo de cada uno de los siguientes temas se introdujeran actividades que afianzaran o permitieran el desarrollo de la competencia comunicativa escrita. 
Si bien en la última etapa de este trabajo de investigación se detectaron insuficiencias en la comunicación escrita de algunos alumnos ya que, por ejemplo, sus producciones aún mostraban falta de coherencia y lógica en la construcción de ideas, el análisis de los resultados permite concluir que la aplicación de esta metodología de trabajo contribuyó notoriamente a mejorar la competencia comunicativa escrita de los estudiantes.

Si bien esta forma de trabajo se aplicó en una sola cátedra, sería conveniente incorporarla en el dictado de todas las asignaturas de cada una de las carreras. Solo así se podrá superar el dicho tan conocido de que "todos saben que los ingenieros no saben escribir". 


\section{REFERENCIAS}

[1] Giordano Lerena, R. \& Cirimelo, S. (2013). Competencias en ingeniería y eficacia institucional. Ingeniería Solidaria, 9 (16), 119 - 127.

[2] Arana Ercilla, M. \& Batista Tejeda, N. (1999). La educación en valores: una propuesta pedagógica para la formación profesional. Pedagogía Universitaria, 4 (3).

[3] Giordano Lerena, R. Compilador (2016) Competencias y perfil del Ingeniero Iberoamericano, formación de profesores y desarrollo tecnológico e innovación. ASIBEI.

[4] Hymes, D.H. (1972). On Communicative Competence. En Pride J. \& Holmes, J. (Ed.) Sociolinguistics, (pp.269-293). Harmondsworth: Penguin.

[5] Bermúdez, L. \& González, L. (2011). La competencia comunicativa: elemento clave en las organizaciones. QUÓRUM ACADÉMICO, 8 (15), 95 - 110.

[6] Caligaris, M.; Rodríguez, G. \& Laugero, L. (2019). La comunicación... ¿una competencia sin importancia en la formación de los ingenieros? Congreso Latinoamericano "Prácticas problemáticas y desafíos contemporáneos de la Universidad y del Nivel Superior", Universidad Nacional de Rosario. Facultad de Humanidades y Artes, Rosario, Argentina.

[7] Gérard, F. \& Roegiers, X. (1993). Concevoir et évaluer les manuels scolaires. Bruxelles: De Boeck-Wesmael. En Roegiers, X. (2007). Pedagogía de la integración: Competencias e integración de los conocimientos en la enseñanza. San José: Coordinación Educativa y Cultural Centroamericana y AECI. Colección IDER (Investigación y desarrollo educativo regional).

[8] Le Boterf, G. (2001). Ingeniería de las competencias. Barcelona: Gedisa.

[9] R. Giordano Lerena, Compilador (2016) Competencias y perfil del Ingeniero Iberoamericano, formación de profesores y desarrollo tecnológico e innovación. ASIBEI.

[10] Labrador, M. \& Morote, P. (2015). La competencia comunicativa en la Universidad. En Celma Valero, M., Gómez del Castillo, M. y Morán Rodríguez, C. (Eds). Memoria del L Congreso Internacional de la Asociación Europea de Profesores de Español, (pp. 360 - 370). Burgos: Universidad Isabel I de Castilla.

[11] Whitin, D. \& Whitin, P. (2002). Promoting communication in the mathematics classroom. Teaching Children Mathematics, 9 (4), 205 - 211.

[12] Silbey, R. (2003). Math out loud! Instructor, 112 (7), 24 - 26.

[13] Flores Aguilar, M. (2014). La competencia comunicativa escrita de los estudiantes de ingeniería y la responsabilidad institucional. Innovación Educativa, 14 (65), 43 - 60.

[14] Medina Cardozo, I. \& Arnao Vásquez, M. (2013). Coherencia y cohesión en el discurso escrito de estudiantes universitarios. Revista de Investigación y Cultura, 2 (1), 44 - 57. 
[15] Kindelán, M. \& Martín, A. (2008). Ingenieros del siglo XXI: importancia de la comunicación y de la formación estratégica en la doble esfera educativa y profesional del ingeniero. Arbor. Ciencia, Pensamiento y Cultura, 184 (732), 731-742. 